TITLE:

\title{
Preparation of polymer-based multimodal imaging agent to visualize the process of bone regeneration.
}

\section{AUTHOR(S):}

Liu, Jian; Jo, Jun-Ichiro; Kawai, Yuko; Aoki, Ichio;

Tanaka, Chuzo; Yamamoto, Masaya; Tabata, Yasuhiko

\section{CITATION:}

Liu, Jian ... [et al]. Preparation of polymer-based multimodal imaging agent to visualize the process of bone regeneration.. Journal of controlled release 2012, 157(3): 398-405

\section{ISSUE DATE:}

2012-02-10

URL:

http://hdl.handle.net/2433/154583

\section{RIGHT:}

@ 2011 Elsevier B.V.; この論文は出版社版でありません。引用の際には 出版社版をご確認ご利用ください。; This is not the published version. Please cite only the published version. 


\section{Preparation of polymer-based multimodal imaging agent to visualize the process of bone regeneration}

Jian Liu ${ }^{1)}$, Jun-ichiro Jo ${ }^{1,2)}$, Yuko Kawai ${ }^{3)}$, Ichio Aoki ${ }^{2)}$, Chuzo Tanaka ${ }^{4)}$, Masaya Yamamoto ${ }^{1)}$, and Yasuhiko Tabata ${ }^{1)}$

Short / Running Title: Multimodal imaging agent for bone regeneration

1) Department of Biomaterials, Institute for Frontier Medical Sciences, Kyoto University, Kyoto 606-8507, Japan

2) Molecular Imaging Center, National Institute of Radiological Sciences, Chiba 263-8555, Japan

${ }^{3)}$ Department of Medical Informatics, Meiji University of Integrative Medicine, Kyoto 629-0392, Japan

4) Department of Neurosurgery, Meiji University of Integrative Medicine, Kyoto, 629-0392, Japan.

Correspondence to Dr. Yasuhiko Tabata, Department of Biomaterials, Field of Tissue Engineering, Institute for Frontier Medical Sciences, Kyoto University, 53 Kawara-cho Shogoin, Sakyo-ku Kyoto 606-8507, Japan.

TEL +81-75-751-4121

FAX $\quad+81-75-751-4646$

E-mail yasuhiko@frontier.kyoto-u.ac.jp 


\begin{abstract}
The objective of this study is to design a new multimodal imaging system for the evaluation of bone regeneration process. Pamidronate (PA) of bisphosphonates with a high affinity for hydroxyapatite, was introduced to pullulan with different molecular weights (PA-pullulan). Then, two probes for fluorescence and magnetic resonance (MR) imagings were introduced into the PA-pullulan to prepare the PA-pullulan conjugates containing both the imaging probes (PA-pullulan-F/M). The PA-pullulan-F/M conjugates had an affinity for hydroxyapatite. A gelatin hydrogel incorporating bone morphogentic protein (BMP)-2 was prepared and implanted subcutaneously into mice to obtain an animal model of bone regeneration. When intravenously injected into mice with the bone tissue ectopically formed by the BMP-2-incorporated hydrogel to fluorescently evaluate their body distribution, the PA-pullulan-F/M conjugates were accumulated in the bone tissue regenerated. The time profile of fluorescent intensity well corresponded with that of calcium amount in the bone tissue newly formed. In addition, the PA-pullulan-F/M conjugates showed an MR ability similar to Gd-DTPA (gadopentetate dimeglumine) of a MR imaging agent clinically used. The MR signal around the bone tissue newly formed was enhanced for mice injected with PA-pullulan-F/M prepared from pullulan with the molecular weight of 6,000 . It is concluded that the PA-pullulan-F/M conjugate is a useful multimodal agent of polymeric delivery system to evaluate the process of bone regeneration.
\end{abstract}




\section{Introduction}

With the recent development of molecular or cell biology and tissue engineering, it has been practically possible to treat diseases based on the natural-healing potential of patients themselves. This is called the therapy of regeneration medicine and has been expected as a new medical treatment. Some animal and clinical trials have been successfully performed to regenerate and repair injured or defection tissues, such as bone, cartilage, skin, and nerve tissues by making use of cells and biomaterials for the drug delivery system (DDS) of growth factors and cell scaffolds [1-6]. However, the technology and methodology to evaluate the extent and process of tissue regeneration and repairing still depend on histological [7], biochemical [8], and morphological examinations [9] of conventional diagnosis. The clinical availability of examinations was limited in terms of their invasiveness and reliability. In addition, they cannot be always applied to observe the time course of change which is important to accurately evaluate the process of tissue regeneration. Under these circumstances, it is necessary to develop a new diagnosis system for the non-invasive evaluation of tissue regeneration in the time sequence fashion.

Noninvasive imaging technologies, such as magnetic resonance imaging (MRI), positron emission tomography (PET), single photon emission computed tomography (SPECT), near-infrared fluorescence (NIRF) imaging, and computed tomography (CT), have been widely used in clinical diagnosis. However, each imaging modality is based on quite different principles and has the advantages and disadvantages. Generally, a single technique does not always correspond to all the requirements for diagnosis imaging [10]. As one trial to tackle the issue, it is practically possible to design a multimodal imaging system. A combinational imaging system composed of different imaging modalities may compensate the deficiencies of single imaging modality, while it gives useful and new tools to biomedical researches and clinical diagnosis. Currently, some prototypes of multimodal imaging system including MRI-optical, NIRF-SPECT, PET-CT, and SPECT-MRI, have been introduced [11-14]. However, the research and development of multimodal imaging probes are still in an early stage although they are highly required to realize the idea of multimodal imaging.

Generally, bone healing and repairing have been diagnosed so far by CT, X-ray, and MRI and their methodology has obtained the clinical reliability. However, the convention imaging methods cannot always provide detailed information about soft and bone tissues regenerated even in the early stage $[15,16]$. Although several researches have been performed on bone-specific imaging probes [17-19], few multimodal imaging probes are developed. If a multimodal imaging system to visualize bone tissue regeneration can be developed, the extent of bone regeneration and repairing will be more 
clearly diagnosed and consequently become a new therapeutic strategy with high reliability. In addition, the system can also give an evaluation method to observe whether or not the process of bone tissue regeneration takes place properly. For this purpose, the modality combination of MRI with a superior property of tomography as well as a high spatial resolution and optical imaging with a high sensitivity and specificity would be a promising choice. To design and prepare such a multimodal imaging system, it is also of prime necessity to make use of DDS technology to enhance the accumulation of imaging agents in the target tissue, resulting in the increased $\mathrm{S} / \mathrm{N}$ ratio.

In this study, pamidronate (PA), which is one type of bisphosphonates with a high affinity for the hydroxyapatite of bone tissues [20,21], was introduced to pullulan with different molecular weights (PA-pullulan). Next, fluorescence and MR imaging probes were introduced to prepare PA-pullulan conjugates containing two imaging probes (PApullulan-F/M). The affinity of PA-pullulan-F/M conjugates for hydroxyapatite and the in vivo fluorescence imaging of bone tissue ectopically formed were evaluated in terms of the molecular weight of pullulan conjugated. The in vitro proton relaxation time and in vivo MR imaging of PA-pullulan-F/M conjugates were also examined.

\section{Materials and methods}

\section{Materials and reagents}

Pullulan with weight-average molecular weights of 6,000, 12,000, and 23,000 were purchased from Hayashibara Biochemical Laboratories, Inc., Okayama, Japan. Pamidronic acid sodium salt (PA) was purchased from Toronto Research Chemicals Inc., North York, ON, Canada. N,N'-carbonyldiimidazole (CDI) and ethylenediamine were purchased from Wako Pure Chemical Industries, Ltd., Osaka, Japan. Hydroxyapatite (HA) beads (Type II, the average diameter $=40 \mu \mathrm{m}$ ) was purchased from Bio-Rad Laboratories, Inc., Hercules, CA. Cy5.5 and Cy5 mono-functional Nhydroxysuccinimidyl esters (Cy5.5-NHS and Cy5-NHS) were purchased from GE Healthcare, Ltd., Buckinghamshire, UK. Bone morphogentic protein (BMP)-2 was kindly obtained from Yamanouchi Pharmaceutical Co., Japan. Diethylenetriaminepentaacetic acid (DTPA) anhydride and 2, 4, 6-Trinitrobezenesulfonic acid (TNBS) were purchased from Sigma-Aldrich Co., St Louis, MO and Nakalai Tesque Inc., Kyoto, Japan, respectively. All the chemicals were used as obtained and without further purification.

\section{Chemical conjugation of pullulan to PA (Scheme1)}

PA was introduced to the hydroxyl groups of pullulan by a CDI activation method [22]. Briefly, various molar amounts of CDI were added to $10 \mathrm{ml}$ of dehydrated dimethylsulfoxide (DMSO) containing $100 \mathrm{mg}$ of pullulan. The molar ratio of CDI initially added to the hydroxyl groups of pullulan was defined as a $[\mathrm{CDI}] /[\mathrm{OH}]$ ratio. 
Following agitation using a magnetic stirrer at room temperature for $3 \mathrm{hr}$, the reaction mixture was precipitated by absolute ethanol. The precipitate was washed several times with absolute ethanol and re-dissolved in $10 \mathrm{ml}$ of $0.1 \mathrm{M}$ carbonate buffer $(\mathrm{pH}$ 9.4) containing $20 \mathrm{mg}$ PA, followed by agitation for $24 \mathrm{hr}$ at room temperature. The reaction mixture was purified by a gel filtration of a PD-10 column (GE healthcare UK ltd., Buckinghamshire, UK) with double-distilled water (DDW), and then freeze-dried to obtain the PA-introduced pullulan (PA-pullulan). The un-reacted PA was determined by the TNBS method [23] to assess the percentage of PA introduced.

\section{Introduction of imaging probes to PA-pullulan}

Ethylenediamine was similarly introduced to the hydroxyl groups of pullulan by the CDI activation method as described above. Briefly, the CDI was added to $10 \mathrm{ml}$ of dehydrated DMSO containing $100 \mathrm{mg}$ of PA-pullulan, followed by the addition of ethylenediamine. Following agitation at room temperature for $24 \mathrm{hr}$, the reaction mixture was precipitated by absolute ethanol, washed with absolute ethanol, and re-dissolved in $10 \mathrm{ml}$ of DDW. After freeze-drying, the aminized PA-pullulan (PA-pullulan- $\mathrm{NH}_{2}$ ) was obtained and the percentage of amino groups introduced was determined by the TNBS method.

The PA-pullulan- $\mathrm{NH}_{2}$ was dissolved in $1 \mathrm{ml}$ of dehydrated DMSO, and then to the DMSO solution, Cy5.5-NHS and DTPA anhydride were added in various addition ratios. After reaction in room temperature for $6 \mathrm{hr}$, the reaction mixture was precipitated by absolute ethanol. The amount of Cy5.5 and DTPA introduced to PA-pullulan- $\mathrm{NH}_{2}$ was determined according to the following procedures. First, the total amount of Cy5.5 and DTPA introduced was calculated by the amount change of amino groups during the introduction of Cy5.5 and DTPA by the TNBS method. Next, the fluorescence intensity of Cy5.5-introduced PA-pullulan- $\mathrm{NH}_{2}$ was measured by Gemini EM fluorescent microplate reader (Molecular Devices, Sunnyvale CA) to obtain the amount of Cy5.5 introduced. Then, the amount of DTPA introduced was calculated by subtracting the amount of Cy5.5 and DTPA introduced from that of Cy5.5. Results were shown the relative molar percentage of Cy5.5 or DTPA, when the amount of Cy5.5 and DTPA was regarded as 100 mole\%. The Cy5.5- and DTPA-introduced PA-pullulan- $\mathrm{NH}_{2}$ was redissolved in DDW, and the solution $\mathrm{pH}$ was adjusted to 6.5 by the addition of $1 \mathrm{~N} \mathrm{NaOH}$ aqueous solution. Then, $100 \mu \mathrm{g}$ of gadolinium acetate was added to the solution, followed by $1 \mathrm{hr}$ leaving for $\mathrm{Gd}^{3+}$ chelation to obtain $\mathrm{Gd}^{3+}$-chelated PA-pullulan-Cy5.5/DTPA (PA-pullulan-F/M). The PA-pullulan-F/M was purified by a PD-10 column with DDW and the amount of $\mathrm{Gd}^{3+}$ ion in each elution fraction was measured by atomic absorption spectrophotometer (AA-6800, Shimadzu Corp., Kyoto,. Japan). For the histology examination, Cy5 introduced PA-pullulan-F/M was prepared under the same conditions except to use Cy5-NHS in place of Cy5.5-NHS. 


\section{Evaluation of binding affinity of PA-pullulan-F/M for hydroxyapatite}

The affinity of PA-pullulan-F/M for HA was evaluated according to the binding experiment reported previously [24, 25]. Briefly, $50 \mathrm{mg}$ HA beads were suspended in 0.5 $\mathrm{ml}$ of saline and mixed with $0.2 \mathrm{ml}$ of PA-pullulan-F/M saline solution. The mixture was placed at room temperature for $30 \mathrm{~min}$ with continuous mixing by vortex $(1,000 \mathrm{rpm}$, Eppendorf thermomixer, Eppendorf AG, Hamburg, Germany), and then centrifuged at 4 ${ }^{\circ} \mathrm{C}$ for $10 \mathrm{~min}$ at 20,000G (Eppendorf centrifuge 5417R, Eppendorf AG, Hamburg, Germany). The supernatant was carefully transferred to each well of 96-multiwell assay plate $\left(\right.$ COSTAR $^{\circledR} 3915$, Corning Inc, Corning, NY) and the fluorescent intensity of unbound PA-pullulan-F/M in the solution form was measured by SpectraMax Gemini EM Fluorescence Microplate reader (Molecular Devices, sunnyvale CA). Each experimental group is composed of 3 samples.

\section{Preparation of mouse model of ectopic bone formation}

A biodegradable hydrogel with a water content of $97.8 \mathrm{wt} \%$ was prepared through the chemical crosslinking of aqueous gelatin solution with glutaraldehyde according to the method described previously [26]. Briefly, $3 \mathrm{wt} \%$ gelatin aqueous solution containing $0.16 \mathrm{wt} \%$ glutaraldehyde was cast into a polypropylene dish $\left(138 \times 138 \mathrm{~mm}^{2}\right.$, Ina Optika Co., Ltd., Osaka Japan), followed by crosslinking reaction at $4^{\circ} \mathrm{C}$ for $12 \mathrm{hr}$. The crosslinked hydrogel prepared was punched out to obtain the discs of 6 $\mathrm{mm}$ in diameter. The hydrogel discs were stirred in $100 \mathrm{mM}$ aqueous glycine solution at $37^{\circ} \mathrm{C}$ for $1 \mathrm{hr}$ to block the residual aldehyde groups of glutaraldehyde. Following washing three times with DDW, the hydrogels were freeze-dried and sterilized with ethylene oxide gas.

To prepare a gelatin hydrogel incorporating BMP-2, $20 \mu \mathrm{l}$ of aqueous solution containing $5 \mu \mathrm{g}$ of BMP-2 was dropped onto the freeze-dried hydrogel, followed by leaving them at $4{ }^{\circ} \mathrm{C}$ overnight. Then, the gelatin hydrogel incorporating BMP-2 was implanted into the back subcutis of ddY mice (male, 6W, Japan SLC, Inc, Shizuoka, Japan) to prepare a mouse model of ectopic bone formation. The mice prepared were used in the following imaging and histochemical studies.

\section{Fluorescence imaging of ectopic bone formation and calcification evaluation}

To fluorescently evaluate the process of bone regeneration in the time-sequential fashion, the PA-pullulan-F/M conjugates prepared from pullulan with molecular weights of $6,000,12,000$, and 23,000 of the same fluorescent intensities were injected intravenously into the mouse model of ectopic bone formation prepared at different time intervals for 4 weeks. The PA-pullulan-F/M was injected to the mice though the tail vein $0,1,2,3$, and 4 weeks after the hydrogel implantation, and $6 \mathrm{hr}$ later, the mice were observed by an optical imaging system (Luminescence analyzer system (LAS) 4000, GE 
healthcare UK ltd., Buckinghamshire, UK). After the fluorescence imaging experiment, the mice were sacrificed and the hydrogel implanted plus the surrounding soft tissues were taken out. The tissues obtained were cut into small pieces and digested in $1 \mathrm{~N} \mathrm{HCl}$ solution in DDW for 1 week at room temperature with occasional mixing. After neutralization with $1 \mathrm{~N} \mathrm{NaOH}$, the supernatant solution was tested by a Calcium $\mathrm{C}$ kit (Wako Pure Chemical Industries, Ltd., Osaka, Japan) to determine the amount of calcium ions as a measure of bone formation. The experimental group is composed of 3 mice.

\section{Histochemical evaluation of bone tissue ectopically formed}

Histological examination was performed for tissue around the hydrogel implanted of mice receiving various PA-pullulan-F/M conjugates. For mice 4 weeks after the hydrogel implantation, PA-pullulan-F/M conjugate was injected intravenously and $6 \mathrm{hr}$ later, the mice were sacrificed and the skin tissue including the hydrogel implanted was taken out. After the confirmation of bone generation by soft X-ray photography, the subcutaneous tissue including the gelatin hydrogel incorporating BMP-2 was embedded into Tissue-Tek (Sakura, Co., Ltd., Aichi, Japan), followed by freezing in liquid nitrogen. After fixation with ethanol, serial cryo-sections of the tissues (5 $\mu \mathrm{m}$ thickness) were prepared for the following staining and microscopic viewing.

The calcium deposition of tissues was visualized by the von Kossa staining according to the method reported by Drury et al. [27]. Briefly, the sections were stained with silver nitrate with the irradiation of UV light for $20 \mathrm{~min}$, followed by washing with sodium thiosulphate and DDW. The sections prepared were counter-stained by nuclear fast red and histologically viewed on a light microscope (AX-80, Olympus, Japan). In addition, the conventional hematoxylin and eosin (H\&E) stain was also performed. On the other hand, the tissue sections of mice receiving the injection of PA-pullulan-F/M conjugate were viewed by using a confocal laser scanning microscopy system (LSM-510, Carl Zeiss, Jena, Germany) for the localization of conjugates while the control experiment was done for the tissue of saline-injected mice.

\section{In vitro and in vivo MRI studies of PA-pullulan-F/M}

The in vitro MRI study was done as described in Supplementary Information. For the in vivo MRI study, the PA-pullulan-F/M was administered to the mice 3 weeks after the hydrogel implantation. The MRI acquisitions were performed in a $7.0 \mathrm{~T}, 40 \mathrm{~cm}$ bore horizontal magnet (Kobelco and Jastec, Kobe, Japan) interfaced to a Bruker Avance console (Bruker Biospin, Ettlingen, Germany) with a volume coil for transmission and reception (35 mm internal diameter, Rapid Biomedical, Rimpar, Germany). Prior to and during the MRI scan, all mice were anaesthetized through a facemask with $2.0 \%$ isoflurane (Mylan Japan, Tokyo, Japan). Polyethylene catheter (PE-10, Becton Dickinson, MD) were placed in the tail vein for injecting drug. Rectal temperature was continuously 
monitored and automatically maintained at $36.5 \pm 0.5^{\circ} \mathrm{C}$ using a warm-air heating system (laboratory made) throughout all experiments. Trans-axial multi-slice $\mathrm{T}_{1}$-weighted MR images $\left(\mathrm{T}_{1} \mathrm{WI}\right)$ were acquired with a spin-echo sequence, $(\mathrm{TR} / \mathrm{TE}=350 / 9.57 \mathrm{~ms}, 4$ slices, slice thickness $=1.0 \mathrm{~mm}$, matrix $=256 \times 256$, FOV $=32.0 \times 32.0 \mathrm{~mm}^{2}$, average $=4$, scan time $=6$ min.). Image reconstruction and analysis were performed using ParaVision (Bruker Biospin) and MRVision (Ver. 1.5, MRVision Co., MA). The experimental group is composed of 3 mice.

\section{Statistical analysis}

All the values were shown as the mean value \pm the standard deviation of the mean. The pairwise comparisons of individual group means were conducted based on the Tukey test. The value of $\mathrm{P}<0.05$ was considered to be statistically significant. 


\section{Results}

\section{Preparation and characterization of PA-pullulan-F/M}

A

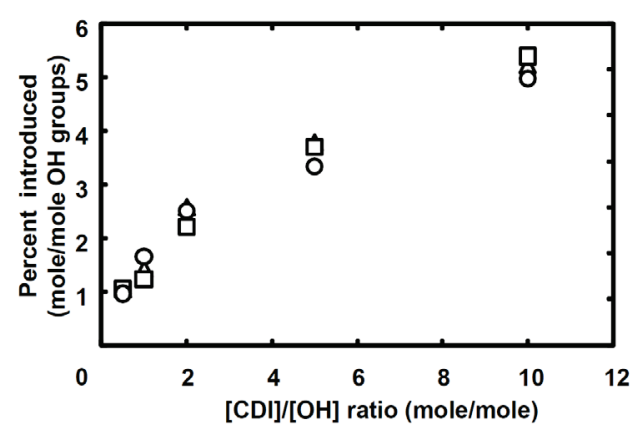

C



B

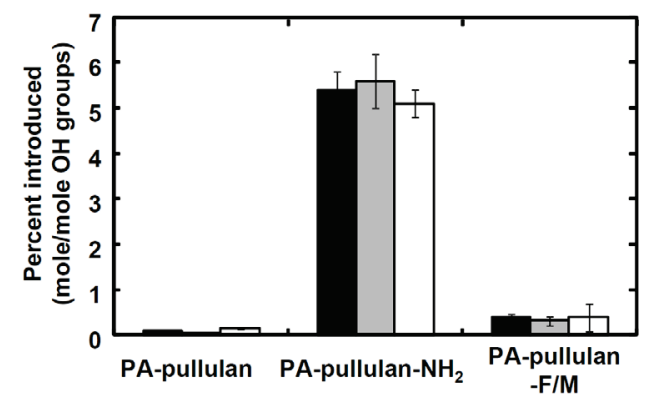

D



Figure 1. A) Effect of the initial addition ratio of $\mathrm{CDI}$ to $\mathrm{OH}$ groups on the percentage of $\mathrm{PA}$ introduced to pullulan with molecular weights of 6,000 (O), 12,000 ( $\triangle$ ), and 23,000 ( $\square$ ). B) Change of amino groups introduced in PA-pullulan before and after the introduction of ethylenediamine (PA-pullulan-NH2) and Cy5.5/DTPA residues of imaging probes (PA-pullulan-Cy5.5/DTPA). The molecular weight of pullulan used is 6,000 (black bars), 12,000 (gray bars) or 23,000 (white bars). The percentage of PA introduced is 3.8 mole\%. B) Percentage of Cy5.5 (white area) and DTPA residues (black area) introduced to PA-pullulan in different ratios of Cy5.5 to DTPA anhydride added initially. The molecular weight of pullulan used is 6,000 . The percentage of PA introduced is 3.8 mole $\%$. C) The elution curve of Gd3 + ions for PA-pullulanF/M prepared by mixing of PA-pullulan-Cy5.5/DTPA and Gd3+ ions. The molecular weight of pullulan used is 6,000 . The percentage of PA introduced is $3.8 \mathrm{~mole} \%$.

PA was introduced to the hydroxyl groups of pullulan by the CDI activation method (Figure 1A). The percentage of PA introduced could be changed by altering the amount of CDI added initially, irrespective of the pullulan molecular weight. Figure 1B shows the change of amino groups introduced in PA-pullulan before and after the introduction of ethylenediamine and Cy5.5/DTPA residues. Ethylenediamine was introduced to the hydroxyl groups of pullulan, while Cy5.5 and DTPA residues were introduced into the amino groups of PA-pullulan. No effect of pullulan molecular weight on the percentage of amino groups and the subsequent Cy5.5 and DTPA residues 
introduced was observed (Figure 1C). The relative ratio of Cy5.5 and DTPA residues introduced to PA-pullulan could be controlled by changing the ratio of Cy5.5-NHS and DTPA anhydride added initially (Figure 1C).

Figure 1D shows the elution curve of $\mathrm{Gd}^{3+}$ ions for mixed solution of PApullulan-Cy5.5/DTPA and $\mathrm{Gd}^{3+}$ ions. Two peaks were detected. One was attributed to $\mathrm{Gd}^{3+}$-chelated PA-pullulan (high molecular weight fraction) and the other was for free $\mathrm{Gd}^{3+}$ ions (low molecular weight fraction). The similar elution pattern was observed for PA-pullulan-Cy5.5/DTPA prepared from pullulan with different molecular weights (date not shown).

\section{Evaluation of binding affinity of PA-pullulan-F/M for hydroxyapatite}

Figure 2 shows the binding profiles of PA-pullulan-F/M with different molecular weights and percentages of PA introduced to hydroxyapatite beads. The binding affinity of PA-pullulan-F/M increased with an increase in the percentage of PA introduced, irrespective of the pullulan molecular weight, whereas PA-free pullulan-F/M had no affinity for hydroxyapatite. When the introduction percentage was less than $3.8 \mathrm{~mole} \%$, the binding affinity of PA-pullulan-F/M prepared from pullulan with the molecular weight of 6,000 was significantly higher than that of PA-pullulan-F/M prepared from pullulan with higher molecular weights. At the PA introduction ratio of 3.8 mole $\%$ or higher, the HA affinity of conjugates reached to a certain level, irrespective of the pullulan molecular weight. Therefore, to investigate the effect of pullulan molecular weight on the imaging efficiency, the percentage of PA introduced was fixed at 3.8 mole $\%$, otherwise mentioned.

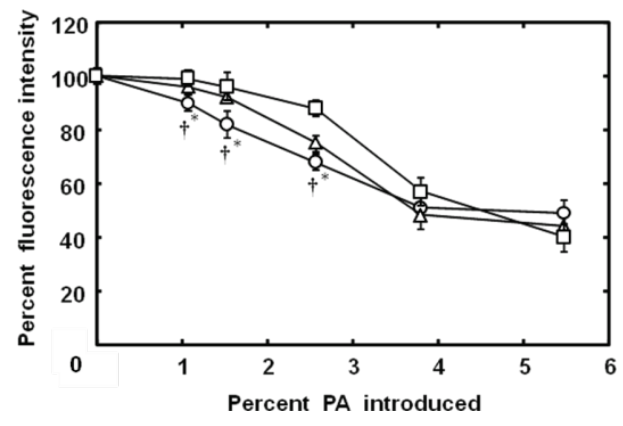

Figure 2. The binding profiles of PA-pullulan-F/M with different percentages of PA introduced to hydroxyapatite beads. The molecular weight of pullulan used is $6,000(\bigcirc), 12,000(\triangle)$ or $23,000(\square)$. The percentage of PA introduced is $3.8 \mathrm{~mole} \%$ and the [Cy5.5]/[DTPA] ratio is $0.1 . *, p<0.05$ : significance against the percent fluorescence of PA-pullulan-F/M prepared from pullulan with the molecular weight of 12,000. $\dagger, \mathrm{p}<0.05$ : significance against the percent fluorescence of PA-pullulan-F/M prepared from pullulan with the molecular weight of 23,000 . 


\section{Bone regeneration imaging by $P$ A-pullulan-F/M}
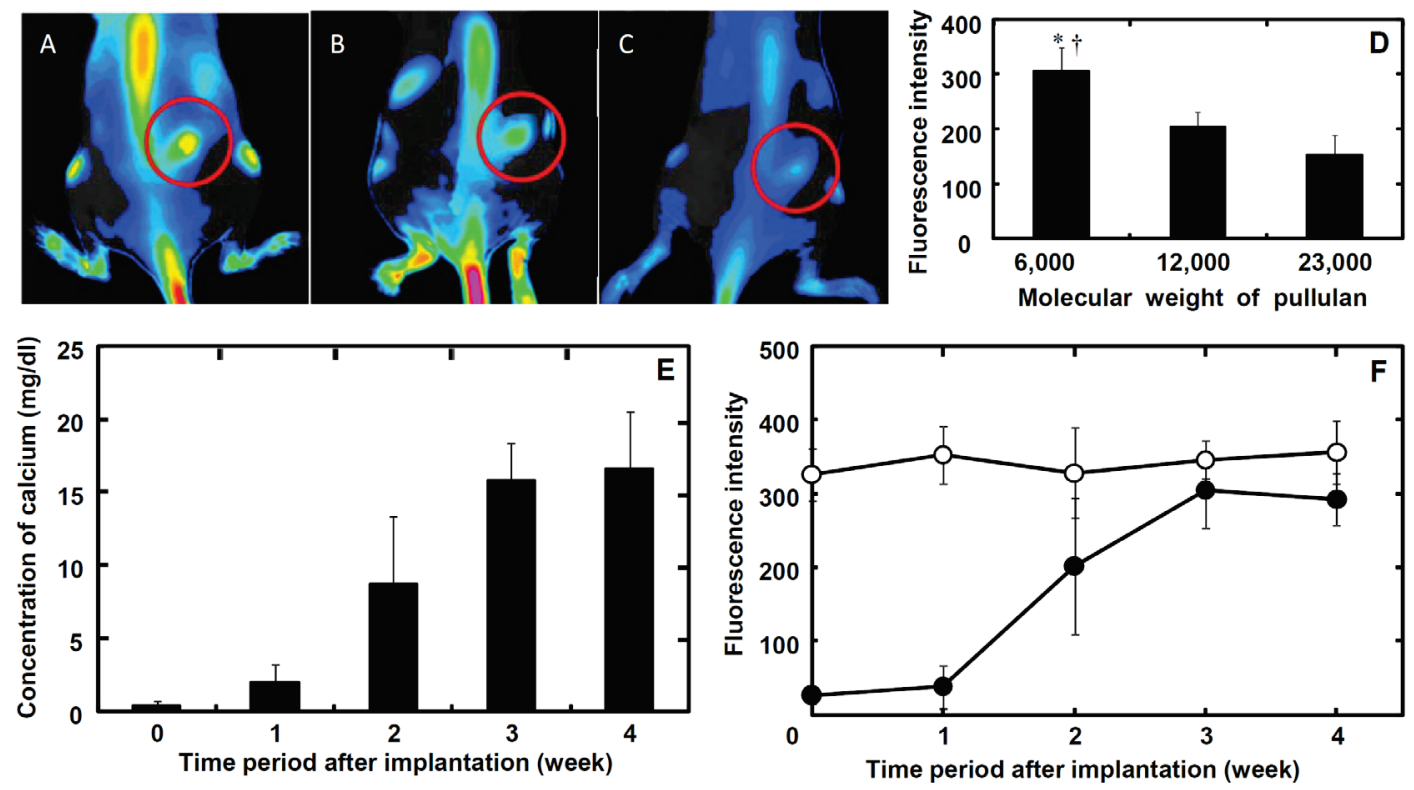

Figure 3. Fluorescence images (A-C) of mice or their intensities of site around the hydrogel incorporating BMP-2 implanted (D) $6 \mathrm{hr}$ after the intravenous injection of PA-pullulan-F/M prepared from pullulan with different molecular weights of 6,000 (A), 12,000 (B), and 23,000 (C). The injection was performed for mice 4 weeks after the hydrogel implantation. The percentage of PA introduced is 3.8 mole $\%$ and the [Cy5.5]/[DTPA] ratio is 0.1. The site implanted with hydrogel incorporating BMP-2 is indicated by a red circle. (D) *, p $<0.05$ : significance against the fluorescence intensity after the injection of PA-pullulan-F/M prepared from pullulan with the molecular weight of 12,000 . $\dagger, p<0.05$ : significance against the fluorescence intensity after the injection of PA-pullulan-F/M prepared from pullulan with the molecular weight of 23,000. E) Time profile of calcium concentration of subcutaneous tissues around gelatin hydrogels incorporating BMP-2 implanted. F) Time profile of fluorescent intensity of normal vertebral bone $(\bigcirc)$ and the site around gelatin hydrogels incorporating BMP-2 implanted (O).

Figures 3A-C shows the fluorescence images of mice $6 \mathrm{hr}$ after the intravenous injection of PA-pullulan-F/M prepared from pullulan with different molecular weights. It is apparent that the PA-pullulan-F/M prepared from pullulan with the molecular weight of 6,000 shows the strongest imaging around the site of bone tissue newly formed. Figure 3D shows the fluorescent intensity of site around the hydrogel incorporating BMP-2 implanted $6 \mathrm{hr}$ after the intravenous injection of PA-pullulan-F/M. The fluorescent intensity for PA-pullulan-F/M with the lowest molecular weight was significantly higher than that of other conjugates.

Figure 3E shows the time profile of calcium concentration around subcutaneous tissues of gelatin hydrogels incorporating BMP-2 implanted. The calcium amount of bone tissue newly formed increased with time to reach a maximum level 3 weeks after implantation of hydrogel. Figure 3F shows the time profiles of fluorescent intensity of vertebral bone and around gelatin hydrogels incorporating BMP-2 implanted. The similar increase pattern was observed for the fluorescent intensity at the bone tissue ectopically formed, while the normal bone tissue showed a constant fluorescent intensity. In addition, 
the fluorescent intensity of bone tissue ectopically formed tended to increase with time to attain that of vertebral bone.

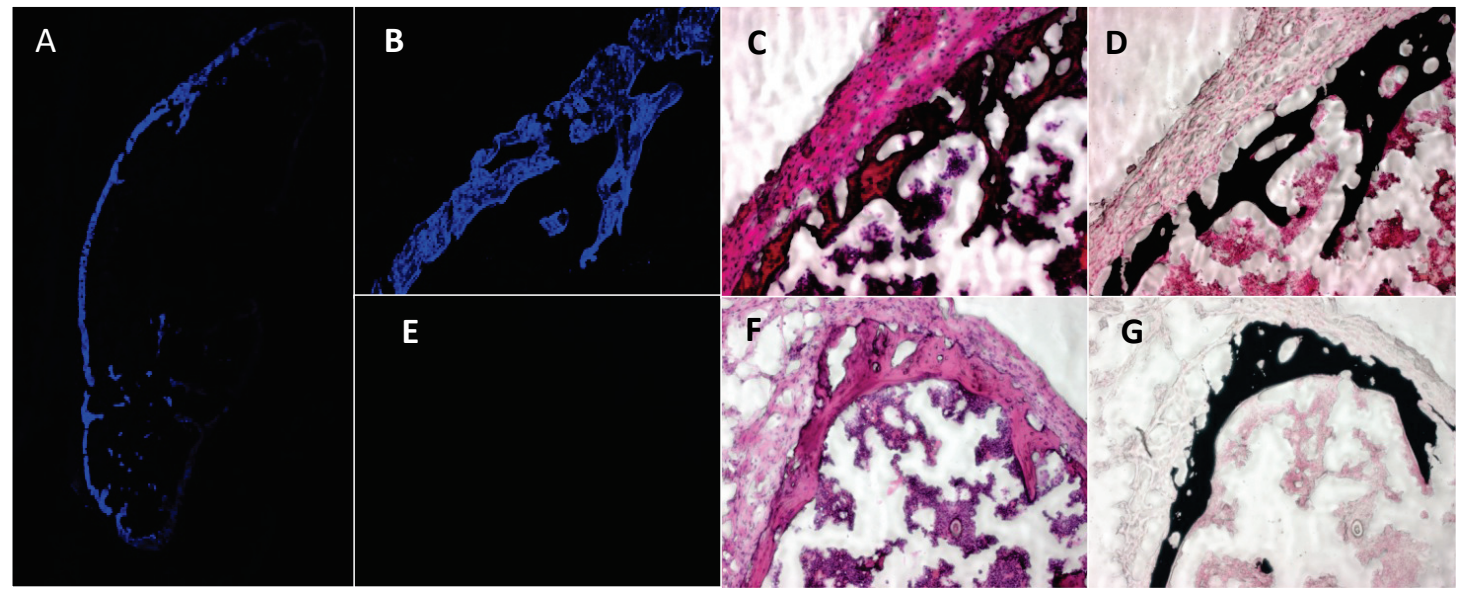

Figure 4. Microscopic images around subcutaneous tissues of gelatin hydrogel incorporating BMP-2 implanted. The tissues were taken out $6 \mathrm{hr}$ after the intravenous injection of PA-pullulan-F/M (A-D) and saline $(\mathrm{E}-\mathrm{G})$ to mice 4 weeks after hydrogel implantation: fluorescence (A, B, and E), H\&E stained (C and F), and von Kossa stained sections (D and $G$ ). The molecular weight of pullulan used is 6,000 . The percentage of PA introduced is $3.8 \mathrm{~mole} \%$ and the [Cy5.5]/[DTPA] ratio is 0.1 .

\section{Histochemical evaluation of bone tissue ectopically formed}

Figure 4 shows the microscopic images of subcutaneous tissues 4 weeks after the implantation of gelatin hydrogel incorporating BMP-2. By using the hydrogel incorporating BMP-2, the bone formation around the hydrogel was observed histochemically. The calcium-deposited tissue area well corresponded to the bone tissue recognized by the H\&E staining. Interestingly, the area of bone tissue newly formed was clearly superposed with that of fluorescence contributing to the PA-pullulan-F/M conjugate intravenously injected. For the saline injection, such a fluorescent imaging was not observed around the site of hydrogel implanted.

\section{In vivo MRI studies of PA-pullulan-F/M}

Figure 5 shows the time profiles of MR images for bone tissue regenerated or muscle tissue of mice injected with PA-pullulan-F/M prepared from pullulan with the molecular weight of 6,000 . The signal of muscle tissue was slightly enhanced and disappeared with time. On the other hand, the signal of bone tissue regenerated was enhanced gradually and retained. 
A

Before

injection

Periods after injection (hr)
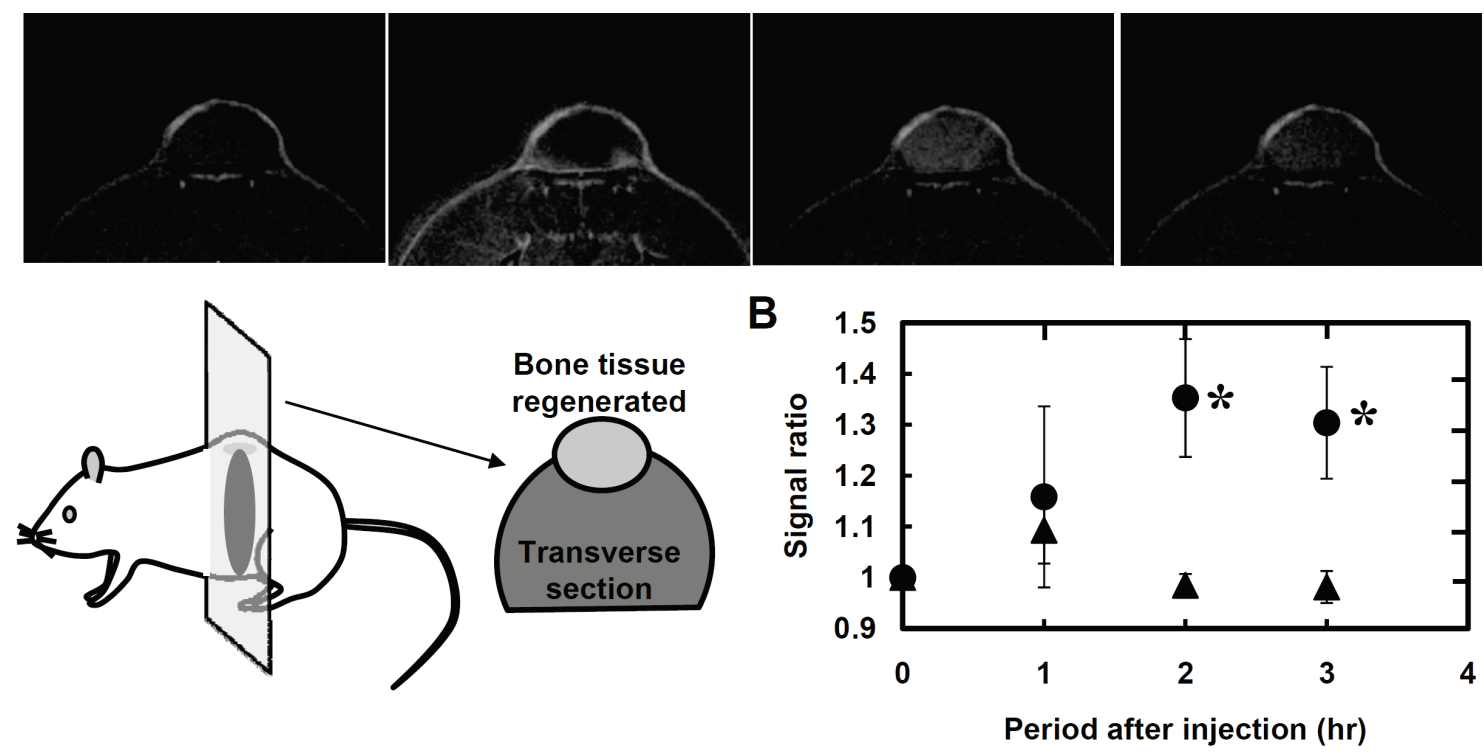

Figure 5. (A) Time profiles of MR images for mice injected with PA-pullulan-F/M. The injection was performed for mice 3 weeks after the hydrogel implantation. Trans-axial multi-slice $\mathrm{T}_{1}$-weighted MR images were repeatedly acquired with a spin-echo sequence, $(\mathrm{TR} / \mathrm{TE}=350 / 9.57 \mathrm{~ms}, 4$ slices, slice thickness $=1.0 \mathrm{~mm}$, matrix $=256 \times 256, \mathrm{FOV}=32.0 \times 32.0 \mathrm{~mm}^{2}$, average $=4$, scan time $=6 \mathrm{~min}$.). (B) Time profiles of MR signal ratio of bone tissue regenerated $(\boldsymbol{O})$ and muscle tissue $(\boldsymbol{\Delta})$ after PA-pullulan-F/M injection. The molecular weight of pullulan used is 6,000. The percentage of PA introduced is $3.8 \mathrm{~mole} \%$ and the [Cy5.5]/[DTPA] ratio is $0.1 .{ }^{*}, \mathrm{p}<0.05$; significance against the MR signal ratio of muscle tissue at the corresponding time.

\section{Discussion}

The main objective of this study is to develop a DDS of non-invasive multimodal imaging for bone regeneration. For this purpose, pamidronate which has a high affinity for the hydroxyapatite of bone tissues was selected as a targeting moiety for bone tissue regenerated. As expected, the pamidronate functioned well to enhance the hydroxyapatite affinity of pullulan. This was experimentally confirmed by the in vitro binding assay (Figure 2) and the in vivo accumulation at the site of bone tissue newly formed (Figures 3,4 , and 5 ).

In the present study, pullulan was used for the polymeric carrier of imaging agent. Pullulan is a water-soluble polysaccharide with a repeated unit of maltotriose condensed through $\alpha-1,6$ linkage. Since the pullulan has a number of hydroxyl groups, several numbers or types of ligands or imaging probes can be simultaneously introduced. Additionally, the modification with a water-soluble polymer enables the ligands or imaging probes to be stably present in the blood circulation $[28,29]$. Therefore, if the 
blood stability of probe is achieved, it is highly expected that the polymer-modified imaging agent enhances the imaging efficacy.

The CDI method was effective in the chemical introduction of PA to pullulan as well as an intermediate derivative of ethylenediamine for the subsequent reaction with fluorescent (Cy5.5 and Cy5) and MR imaging probes (Figures 1A and B). It is apparent from Figure 1B that most of amino groups introduced were converted to the imaging probes and the relative ratio could be controlled by changing the ratio of imaging probes added initially (Figure 1C). It is well known that the detection sensitivity of fluorescence probes is much higher than that of MRI probes [30, 31]. However, for the combination imaging system, it is highly conceivable that the concentration of every probe in the target site to show the imaging activity can be anticipated based on the imaging sensitivity. Therefore, in this study, the [Cy5.5]/[DTPA] ratio of 0.1 was selected (Figure 1C). It is no doubt that the ratio may affect the multimodal imaging ability. Further investigation should be done to optimize the sensitivity for a fluorescence-MR combination multimodal imaging.

The percentage of PA introduced and the molecular weight of pullulan affected the binding affinity of PA-pullulan-F/M conjugates for hydroxyapatite (Figure 2). When the amount of PA conjugated in one pullulan molecule become large, it is possible that the binding site of conjugates to hydroxyapatite increases, resulting an enhanced affinity of PA-pullulan-F/M. However, when the percent PA introduced was 3.8 mole $\%$ or higher, the binding affinity of PA-pullulan F/M became constant. It is apparent that the PA introduction increased with the $[\mathrm{CDI}] /[\mathrm{OH}]$ ratio (Figure 1A). Taken together, this finding indicates that the PA density contributing to the binding affinity has a threshold. The molecular weight of pullulan also affected the binding affinity of PA-pullulan-F/M. When the percentage of PA introduced was low, an increase in the molecular weight of pullulan decreased the binding affinity of PA-pullulan-F/M conjugates. However, at the higher percentage of PA introduced, the affinity became a similar level, irrespective of the molecular weight of pullulan. The reason may be explained by the steric hindrance of pullulan molecules. Longer chains of pullulan with higher molecular weight may result in many and long loops of polymer chains which cause steric hindrance to decrease the contact probability of PA with hydroxyapatite at the low percentage of PA introduced. On the other hand, when the percentage increased, the steric hindrance will be no longer essential and consequently the binding affinity was similar for any PA-pullulan-F/M conjugate. To achieve a high targetability to bone tissues, higher PA introduction ratio may be favorable. However, an excess of PA may cause some side effects because PA itself also has bioactivity $[32,33]$. For these reasons, a balanced PA introduction ratio is needed. Considering that the PA introduction ratio higher than $3.8 \mathrm{~mol} \%$ do not 
contribute to their binding activity to HA any more, the percentage of PA introduced was selected at 3.8 mole $\%$ for all experiments.

For the in vivo fluorescent imaging, the PA-pullulan-F/M prepared from pullulan with the molecular weight of 6,000 showed significantly higher imaging ability than the others (Figures 3A-D). Therefore, further in vivo imaging experiments were performed by using this conjugate. There are two mechanisms that enables the PA-pullulan-F/M to accumulate at the bone tissue regenerated through the extravazation (from blood to interstitial space) and penetration (in the interstitial space to reach the target cells); (1) size of junctions (or channels) in the endoethelial cell layer or of extracellular matrix network size and (2) diffusion-controlled extravazation and penetration. The molecular weight of PA-pullulan-F/M affects both the mechanisms. On one hand, it is highly conceivable that the PA-pullulan-F/M prepared from pullulan with the low molecular weight has a smaller molecular size, which allows the conjugate to reach the bone tissue regenerated. It is well known that the new formation of blood vessels needs the formation of bone tissue [34]. The process is normally accompanied with inflammation reactions [35]. Considering the EPR effect [36] of blood vessels formed in inflammation, it is possible that the conjugate of small size tends to accumulate to a high extent compared with that of too large size. This is one of the reasons why the bone tissue accumulation of PA-pullulan-F/M conjugates had an influence on the molecular weight of pullulan. This type of molecular size selection by the wall of blood capillaries may take place around the bone tissue newly formed by BMP-2. In addition, the bone tissue composed of HA and organic substances may have a complex structure where the large-size imaging agent cannot reach to the HA component of tissue. On the other hand, it is well recognized that the diffusion of substances is governed by the molecular weight [37]. However, at present which mechanism is the main factor to explain the body distribution of PA-pulllulan-F/M is unclear. Further investigation is needed to make clear the mechanisms of molecular weight dependence.

After the implantation of gelatin hydrogel incorporating BMP-2 to the subcutis of mice, the ectopic bone regeneration was achieved. The ectopic model was selected in this study because bone tissue newly formed can be readily recognized histologically. We have demonstrated that the controlled release of BMP-2 effectively induced ectopically bone formation [2]. The calcium assay revealed that bone tissue was formed over the time period of 4 weeks (Figure 3E). The similar time profile was observed for fluorescent intensity of PA-pullulan-F/M accumulated. The good correspondence of time profile between the calcium amount and fluorescent intensity strongly indicates the possibility that the progress of bone regeneration can be fluorescently evaluated by the PA-pullulanF/M. This is also confirmed by histological examinations (Figure 4). The area of calcium deposited which is confirmed by the von Kossa and H\&E staining overlayed that of 
fluorescence emission from the PA-pullulan-F/M. For the saline injection, no fluorescence was detected at the area of calcium deposited. Taken together, it is clearly that the PA-pullulan-F/M was specifically bound to the calcium in the bone tissue regenerated, resulting in the achievement of fluorescence imaging thereat. The PApullulan-F/A had a high MRI contrast enhancement capability similar to the Gd-DTPA (gadopentetate dimeglumine, Magnevist ${ }^{\circledR}$ ) of clinical use (Supplementary table 1). Additionally, the in vivo MR imaging also revealed that the PA-pullulan-F/M was accumulated for the bone tissue regenerated (Figure 5). These findings clearly demonstrate that the PA-pullulan-F/M conjugate is a useful multimodal agent of polymeric delivery system to evaluate the process of bone regeneration. It is well known that DTPA has a high chelate stability to many metal ions, this character make it possible to readily introduce another radioactive isotope for the scintigraphy, PET and SPECT imaging. Some researches to check the possibilities are now under investigation.

\section{Acknowledgements}

The authors would like to thank Ms. Sayaka Shibata, Ms. Chinami Kajiwara, Ms. Aiko Sekita, Mr. Nobuhiro Nitta, and Mr. Teppei Nakahara (National Institute of Radiological Sciences, Japan) for technical assistance.

\section{References}

[1] Y. Tabata, S. Hijikata, M. Muniruzzaman, Y. Ikada, Neovascularization effect of biodegradable gelatin microspheres incorporating basic fibroblast growth factor. J Biomater Sci Polym Ed 10(1) (1999) 79-94.

[2] M. Yamamoto, Y. Takahashi, Y. Tabata, Controlled release by biodegradable hydrogels enhances the ectopic bone formation of bone morphogenetic protein. Biomaterials 24(24) (2003) 4375-4383.

[3] M. Itoh, Y. Hiraoka, K. Kataoka, N.H. Huh, Y. Tabata, H. Okochi, Novel collagen sponge reinforced with polyglycolic acid fiber produces robust, normal hair in murine hair reconstitution model. Tissue Eng 10(5-6) (2004) 818-824.

[4] K. Kojima, R.A. Ignotz, T. Kushibiki, K.W. Tinsley, Y. Tabata, C.A. Vacanti, Tissue-engineered trachea from sheep marrow stromal cells with transforming growth factor beta 2 released from biodegradable microspheres in a nude rat recipient. J Thorac Cardiovasc Surg 128(1) (2004) 147-153.

[5] S. Bajada, I. Mazakova, J.B. Richardson, N. Ashammakhi, Updates on stem cells and their applications in regenerative medicine. J Tissue Eng Regen Med 2(4) (2008) 169-183. 
[6] A. Marui, Y. Tabata, S. Kojima, M. Yamamoto, K. Tambara, T. Nishina, Y. Saji, K. Inui, T. Hashida, S. Yokoyama, R. Onodera, T. Ikeda, M. Fukushima, M. Komeda, A novel approach to therapeutic angiogenesis for patients with critical limb ischemia by sustained release of basic fibroblast growth factor using biodegradable gelatin hydrogel: an initial report of the phase I-IIa study. Circ J 71(8) (2007) 1181-1186.

[7] K. Lysiak-Drwal, M. Dominiak, L. Solski, B. Zywicka, S. Pielka, T. Konopka, H. Gerber, Early histological evaluation of bone defect healing with and without guided bone regeneration techniques: experimental animal studies. Postepy Hig Med Dosw (Online) 62 (2008) 282-288.

[8] E. Daglioglu, M.S. Dike, K. Kilinc, D. Erdogan, G. Take, F. Ergungor, O. Okay, Z. Biyikli, Neuroprotective effect of melatonin on experimental peripheral nerve injury: an electron microscopic and biochemical study. Central European neurosurgery. Central European neurosurgery 70(3) (2009) 109-114.

[9] X.B. Fu, T.Z. Sun, X.K. Li, Z.Y. Sheng, Morphological and distribution characteristics of sweat glands in hypertrophic scar and their possible effects on sweat gland regeneration. Chin Med J (Engl) 118(3) (2005) 186-191.

[10] T.F. Massoud, S.S. Gambhir, Molecular imaging in living subjects: seeing fundamental biological processes in a new light. Genes Dev 17(5) (2003) 545-580.

[11] K. Chtourou, M. Maloul, F. Kallel, S. Charfedine, F. Hamza, F. Guermazi, SPECT and MRI fusion for an extended bilateral osteonecrosis. Eur J Nucl Med Mol Imaging 35(12) (2008) 2343.

[12] V. Ntziachristos, A.G. Yodh, M. Schnall, B. Chance, Concurrent MRI and diffuse optical tomography of breast after indocyanine green enhancement. Proc Natl Acad Sci U S A 97(6) (2000) 2767-2772.

[13] T. Beyer, D.W. Townsend, T. Brun, P.E. Kinahan, M. Charron, R. Roddy, J. Jerin, J. Young, L. Byars, R. Nutt, A combined PET/CT scanner for clinical oncology. J Nucl Med 41(8) (2000) 1369-1379.

[14] O. Veiseh, C. Sun, J. Gunn, N. Kohler, P. Gabikian, D. Lee, N. Bhattarai, R. Ellenbogen, R. Sze, A. Hallahan, J. Olson, M. Zhang, Optical and MRI multifunctional nanoprobe for targeting gliomas. Nano Lett 5(6) (2005) 1003-1008.

[15] D. Berthoty, P. Haghighi, D.J. Sartoris, D. Resnick, Osseous invasion by softtissue sarcoma seen better on MR than on CT. AJR Am J Roentgenol 152(5) (1989) 1131. [16] J.J. Peterson, L.W. Bancroft, M.J. Kransdorf, Principles of bone and soft tissue imaging. Hand Clin 20(2) (2004) v, 147-166.

[17] J. de Boer, C. van Blitterswijk, C. Lowik, Bioluminescent imaging: emerging technology for non-invasive imaging of bone tissue engineering. Biomaterials 27(9) (2006) 1851-1858. 
[18] K.M. Kozloff, R. Weissleder, U. Mahmood, Noninvasive optical detection of bone mineral. J Bone Miner Res 22(8) (2007) 1208-1216.

[19] D.H. Kempen, M.J. Yaszemski, A. Heijink, T.E. Hefferan, L.B. Creemers, J. Britson, A. Maran, K.L. Classic, W.J. Dhert, L. Lu, Non-invasive monitoring of BMP-2 retention and bone formation in composites for bone tissue engineering using SPECT/CT and scintillation probes. J Control Release 134(3) (2009) 169-176.

[20] D. Wang, S.C. Miller, P. Kopeckova, J. Kopecek, Bone-targeting macromolecular therapeutics. Adv Drug Deliv Rev 57(7) (2005) 1049-1076.

[21] R. Graham, G. Russell, Determinants of structure-function relationships among bisphosphonates. Bone 40 (2007) S21-S25.

[22] G.T. Hermanson, Bioconjugate techniques, Academic Press, 1996.

[23] T.G.M. Schalkhammer, Analytical biotechnology Springer, 2002.

[24] H. Uludag, N. Kousinioris, T. Gao, D. Kantoci, Bisphosphonate conjugation to proteins as a means to impart bone affinity. Biotechnol Prog 16(2) (2000) 258-267.

[25] M.F. Jarvis, C.J. Burns, H.W. Pauls, A. Assal, J.S. Kim, D.L. Cheney, R.D. Youssefyeh, Characterization of the bisphosphonate recognition site on hydroxyapatite using radioligand binding techniques with [14C]citric acid. Calcif Tissue Int 52(5) (1993) 372-377.

[26] M. Yamamoto, Y. Tabata, L. Hong, S. Miyamoto, N. Hashimoto, Y. Ikada, Bone regeneration by transforming growth factor beta1 released from a biodegradable hydrogel. J Control Release 64(1-3) (2000) 133-142.

[27] R. Drury, E. Wallington, Demonstration of calcium salts. In: Carleton's Histological Techniques, 5th ed., Oxford University Press, 1980.

[28] R. Duncan, The dawning era of polymer therapeutics. Nat Rev Drug Discov 2(5) (2003) 347-360.

[29] P.A. Vasey, S.B. Kaye, R. Morrison, C. Twelves, P. Wilson, R. Duncan, A.H. Thomson, L.S. Murray, T.E. Hilditch, T. Murray, S. Burtles, D. Fraier, E. Frigerio, J. Cassidy, Phase I clinical and pharmacokinetic study of PK1 [N-(2hydroxypropyl)methacrylamide copolymer doxorubicin]: first member of a new class of chemotherapeutic agents-drug-polymer conjugates. Cancer Research Campaign Phase I/II Committee. Clin Cancer Res 5(1) (1999) 83-94.

[30] V.M. Runge, J.W. Wells, Update: safety, new applications, new MR agents. Top Magn Reson Imaging 7(3) (1995) 181-195.

[31] W. Mason, Fluorescent and luminescent probes for biological activity, Academic Press, London, 1999.

[32] M.C. Lodder, P.A. Van Pelt, W.F. Lems, P.J. Kostense, C.H. Koks, B.A. Dijkmans, Effects of high dose IV pamidronate on disease activity and bone metabolism 
in patients with active RA: a randomized, double-blind, placebo-controlled trial. J Rheumatol 30(9) (2003) 2080-2081.

[33] E. Terpos, J. Palermos, K. Tsionos, K. Anargyrou, N. Viniou, P. Papassavas, J. Meletis, X. Yataganas, Effect of pamidronate administration on markers of bone turnover and disease activity in multiple myeloma. Eur J Haematol 65(5) (2000) 331-336.

[34] L.R. Amir, A.G. Becking, A. Jovanovic, F.B. Perdijk, V. Everts, A.L. Bronckers, Formation of new bone during vertical distraction osteogenesis of the human mandible is related to the presence of blood vessels. Clin Oral Implants Res 17(4) (2006) 410-416.

[35] C.H. Rundle, H. Wang, H. Yu, R.B. Chadwick, E.I. Davis, J.E. Wergedal, K.H. Lau, S. Mohan, J.T. Ryaby, D.J. Baylink, Microarray analysis of gene expression during the inflammation and endochondral bone formation stages of rat femur fracture repair. Bone 38(4) (2006) 521-529.

[36] H. Maeda, J. Wu, T. Sawa, Y. Matsumura, K. Hori, Tumor vascular permeability and the EPR effect in macromolecular therapeutics: a review. J Control Release 65(1-2) (2000) 271-284.

[37] R.K. Jain, Physiological barriers to delivery of monoclonal antibodies and other macromolecules in tumors. Cancer Res 50(3 Suppl) (1990) 814s-819s.

\section{Figure legends}

Figure 1. A) Effect of the initial addition ratio of $\mathrm{CDI}$ to $\mathrm{OH}$ groups on the percentage of PA introduced to pullulan with molecular weights of $6,000(\bigcirc), 12,000(\triangle)$, and 23,000 ( $\square$ ). B) Change of amino groups introduced in PA-pullulan before and after the introduction of ethylenediamine (PA-pullulan-NH2) and Cy5.5/DTPA residues of imaging probes (PA-pullulan-Cy5.5/DTPA). The molecular weight of pullulan used is 6,000 (black bars), 12,000 (gray bars) or 23,000 (white bars). The percentage of PA introduced is 3.8 mole\%. B) Percentage of Cy5.5 (white area) and DTPA residues (black area) introduced to PA-pullulan in different ratios of Cy5.5 to DTPA anhydride added initially. The molecular weight of pullulan used is 6,000 . The percentage of PA introduced is $3.8 \mathrm{~mole} \%$. C) The elution curve of $\mathrm{Gd} 3+$ ions for PA-pullulan-F/M prepared by mixing of PA-pullulan-Cy5.5/DTPA and Gd3+ ions. The molecular weight of pullulan used is 6,000 . The percentage of PA introduced is $3.8 \mathrm{~mole} \%$.

Figure 2. The binding profiles of PA-pullulan-F/M with different percentage of PA introduced to hydroxyapatite beads. The molecular weight of pullulan used is $6,000(\bigcirc)$, $12,000(\triangle)$ or $23,000(\square)$. The percentage of PA introduced is $3.8 \mathrm{~mole} \%$ and the [Cy5.5]/[DTPA] ratio is $0.1 .^{*}, \mathrm{p}<0.05$ : significance against the percent fluorescence of PA-pullulan-F/M prepared from pullulan with the molecular weight of 12,000 . $\uparrow, p<0.05$ : significance against the percent fluorescence of PA-pullulan-F/M prepared from pullulan with the molecular weight of 23,000. 
Figure 3. Fluorescence images (A-C) of mice or their intensities of site around the hydrogel incorporating BMP-2 implanted (D) $6 \mathrm{hr}$ after the intravenous injection of PApullulan-F/M prepared from pullulan with different molecular weights of 6,000 (A), 12,000 (B), and 23,000 (C). The injection was performed for mice 4 weeks after the hydrogel implantation. The percentage of $\mathrm{PA}$ introduced is $3.8 \mathrm{~mole} \%$ and the [Cy5.5]/[DTPA] ratio is 0.1 . The site implanted with hydrogel incorporating BMP-2 is indicated by a red circle. (D) *, p $<0.05$ : significance against the fluorescence intensity after the injection of PA-pullulan-F/M prepared from pullulan with the molecular weight of $12,000 . \dagger, p<0.05$ : significance against the fluorescence intensity after the injection of PA-pullulan-F/M prepared from pullulan with the molecular weight of 23,000. E) Time profile of calcium concentration of subcutaneous tissues around gelatin hydrogels incorporating BMP-2 implanted. F) Time profile of fluorescent intensity of normal vertebral bone $(\bigcirc)$ and the site around gelatin hydrogels incorporating BMP-2 implanted (O).

Figure 4. Microscopic images of subcutaneous tissue sections around gelatin hydrogel incorporating BMP-2 implanted. The tissues were taken out $6 \mathrm{hr}$ after the intravenous injection of PA-pullulan-F/M (A-D) and saline (E-G) to mice 4 weeks after hydrogel implantation: fluorescence (A, B and E), H\&E stained (C and F), and von Kossa stained sections ( $\mathrm{D}$ and $\mathrm{G})$. The molecular weight of pullulan used is 6,000 . The percentage of PA introduced is $3.8 \mathrm{~mole} \%$ and the [Cy5.5]/[DTPA] ratio is 0.1 .

Figure 5. (A) Time profiles of MR images for mice injected with PA-pullulan-F/M. The injection was performed for mice 3 weeks after the hydrogel implantation. Trans-axial multi-slice $\mathrm{T}_{1}$-weighted $\mathrm{MR}$ images were repeatedly acquired with a spin-echo sequence, $(\mathrm{TR} / \mathrm{TE}=350 / 9.57 \mathrm{~ms}, 4$ slices, slice thickness $=1.0 \mathrm{~mm}$, matrix $=256 \times 256, \mathrm{FOV}=$ $32.0 \times 32.0 \mathrm{~mm} 2$, average $=4$, scan time $=6 \mathrm{~min}$.). (B) Time profiles of MR signal ratio of bone tissue regenerated $(\mathbf{O})$ and muscle tissue $(\boldsymbol{\Delta})$ after PA-pullulan-F/M injection. The molecular weight of pullulan used is 6,000 . The percentage of PA introduced is 3.8 mole $\%$ and the $[\mathrm{Cy} 5.5] /[\mathrm{DTPA}]$ ratio is $0.1{ }^{*}, \mathrm{p}<0.05$; significance against the MR signal ratio of muscle tissue at the corresponding time. 